\title{
Perceptions of Healthcare Clinicians on Occupational Therapists Providing Services During Well-Baby Visits
}

\author{
Alexis Brenner \\ Moravian College \\ Lauren Kresge ( $\square$ lkresge2234@gmail.com ) \\ Moravian College \\ Trisha MacLeod \\ Moravian College \\ Rebecca McMaster \\ Moravian College
}

\section{Research Article}

Keywords: occupational therapy, primary care, developmental milestones, well-baby, preventive education, clinicians

Posted Date: December 17th, 2020

DOI: https://doi.org/10.21203/rs.3.rs-125935/v1

License: (c) (i) This work is licensed under a Creative Commons Attribution 4.0 International License. Read Full License 
Perceptions of Healthcare Clinicians on Occupational Therapists Providing Services During Well-Baby Visits

Alexis Brenner, Lauren Kresge, Trisha MacLeod, and Rebecca McMaster

Department of Rehabilitation Sciences: Occupational Therapy

Moravian College

1200 Main Street Bethlehem, PA 18018 United States 


\begin{abstract}
Background: To explore the perceptions of healthcare clinicians on the involvement of occupational therapists during a well-baby visit to allot for more face-to-face time with a healthcare practitioner and more efficient developmental milestone screening. Due to the high demand of healthcare clinicians, well-baby visits are becoming shorter caused by lack of availability for healthcare professionals to take on the roles of parent education. Occupational therapy practitioners are qualified as developmental specialists. Therefore, occupational therapy practitioners' involvement in well-baby visits can compensate for this lack of time and allow for additional parent education and screening for developmental delay. Methods: A 13 question survey to quantitatively analyze the perceptions of healthcare clinicians using a Likert-type psychometric rating scale. The Qualtrics XM survey was distributed to licensed practitioners (i.e. primary care physicians, pediatricians, obstetrician-gynecologists, advanced practice clinicians) through Facebook groups and snowball sampling. Data was analyzed using the Statistical Package for the Social Sciences. Results: Majority of clinicians believed the inclusion of occupational therapy during a well-baby visit would be beneficial in providing preventative education and developmental guidance to prevent developmental delay. This research was successful in determining that gender, age, and years of experience did not influence the perceptions of healthcare clinicians in regard to the inclusion of occupational therapy practitioners in primary care. Additional findings indicated that there is a discrepancy between the needs of parents and caregivers and the perceptions of healthcare clinicians regarding the sufficiency of education and resources provided during well-baby visits. Conclusion: Majority of clinicians believed the inclusion of occupational therapy practitioners during a well-baby visit would be beneficial to provide preventative education and developmental guidance to prevent developmental delay. Gender, age, and years of experience did not influence the perceptions of healthcare clinicians in regard to the inclusion of occupational therapy practitioners in primary care. Trial Registration: Retrospectively registered
\end{abstract}

Keywords: occupational therapy, primary care, developmental milestones, well-baby, preventive education, clinicians 


\section{Declaration}

\section{Abbreviations}

EI: early intervention; OT: occupational therapy; OTP: occupational therapy practitioner; OTPF: occupational therapy practice framework; OBGYN: obstetrician-gynecologist; PCP: primary care physician

\section{Acknowledgements}

We thank the administrators of Facebook groups, all survey participants, and Moravian College Occupational Therapy faculty for their guidance, especially our research mentor, Dr. Sonja Burmeister for their contribution to this research.

\section{Authors' contributions}

$\mathrm{AB}$ made substantial contributions to conception and design of figures and writing of the manuscript. SB oversaw all student researchers, provided comments and revisions to the final manuscript, ensured all survey questions targeted the research question, and distributed surveys. LK made substantial contributions in creation of the survey and data analysis. RM made substantial contributions in review of the literature and connection back to research questions and hypotheses making for a concise and focused study. TM made substantial contributions in interpreting the data and reviewed the draft critically for important content. All authors read and approved the final manuscript.

Correspondence to 1kresge2234@gmail.com.

\section{Funding}

There were no sources of funding for this work.

\section{Availability of data and materials}

The datasets used and analyzed during the current study are available from the corresponding author on reasonable request.

\section{Ethics approval and consent to participate}

This study was approved by Moravian College Institutional Review Board (IRB). The IRB number was 20-0028. All participants were provided informed consent prior to completing the surveys. All methods were carried out in accordance with relevant guidelines and regulations.

\section{Consent for publication}

Not applicable.

\section{Competing interests}

The authors declare that they have no competing interests. 


\section{Background}

Parents and caregivers of newborns and infants rely on the expertise of healthcare clinicians, such as primary care physicians (PCP), pediatricians, obstetrician-gynecologist (OBGYN), and other advanced practice clinicians, to provide guidance about their child's earliest developmental stages. The evolution of pediatric medical care has resulted in shorter patient visits, leaving new parents and caregivers wanting more information regarding infant health, development, and preventative measures. A well-baby visit is defined as a routine check-up performed by a healthcare clinician that occurs at regular intervals per guidance of the American Academy of Pediatrics. Well-baby visits ensure infant health and development during the perinatal period and infancy period. The perinatal period is defined as "starting at 22 weeks of gestation and ending seven completed days after birth" [1]. Following the perinatal period is the period of infancy which spans from prenatal to nine months of age per the periodicity schedule from the American Academy of Pediatrics. The focus of this research highlights the perinatal and infancy periods while assessing parent and caregiver education during well-baby visits, preventative measures for developmental delay, and integration of occupational therapy (OT) as a part of the interprofessional team during a well-baby visit.

Due to the high demand of healthcare clinicians, well-baby visits are becoming shorter caused by a lack of opportunity for healthcare clinicians to effectively provide parent education and inadequate reimbursement methods for these services [2]. Pediatricians have reported limited staff to complete developmental screenings as well as a lack of confidence in healthcare clinicians' abilities to appropriately screen children. Specifically, in 2016, it was reported that time was the number one limitation in terms of screening children for developmental delays [2] Additionally, a direct correlation was identified between the increased frequency of 
developmental screenings and the number of referrals to early intervention (EI) services with the primary diagnosis of developmental delay []ㅡ. EI services primarily provide rehabilitative therapy to patients from birth to age three, inclusive of the perinatal and infancy periods. EI has been found to provide significant benefits to children with developmental delay and allows for parent support by providing techniques to help support children in daily life []ㅡ.

While well-baby visits vary in longevity based on the patient's individual needs, researchers concluded that the majority of parents experienced well-baby visits that took 20 minutes or less [4]. A correlation was found between the duration of the visit and the amount of information being disseminated by the healthcare practitioner including information regarding preventative content [4]. Lack of caregiver knowledge about child development, coupled with the possibility of health care providers misinterpreting or not observing delays during the well-baby visit could hinder the patient's ability to receive critical services and guidance needed in the earliest stages [ㄷ].

As a clinician, it is crucial to provide new parents and caregivers with adequate information regarding their child's development, as many parents and caregivers have reported a lack of preparedness and understanding. Parents are seeking more knowledge and information regarding their baby's development in addition to what is currently being provided []ㅡ. More time for discussion about developmental progress allows parents and caregivers the opportunity to potentially identify developmental delays that may be present during the perinatal and infancy periods.

Occupational therapy practitioners (OTPs) are highly educated, trained, and prepared to assess developmental milestones in the perinatal and infancy populations []. The American Occupational Therapy Association []] outlined education and training as types of interventions 
within the OT scope of practice through the Occupational Therapy Practice Framework (OTPF). Through these intervention methods, OTPs ensure client understanding of task breakdown in order to support their daily occupations and life roles []]. According to the OTPF, the occupation of child rearing includes continual guidance and care to support the needs of a child. OTPs are considered developmental specialists in the aspects of assessing developmental milestones, as well as preparing and educating expectant mothers on typical infant development []․

The purpose of this study was to research current status of clinicians' understanding of the OT scope of practice, specifically, the qualifications that OTPs' have as developmental specialists. This research explored the current content of well-baby visits with healthcare clinicians, how this content relates to prevention for developmental delays, and how OT can be of benefit in the primary care setting. The following research question was proposed: What are the perceptions of healthcare clinicians (PCPs, pediatricians, OBGYNs, and advanced practice clinicians) regarding OTPs providing preventative education and developmental guidance to parents and caregivers during their scheduled well-baby visits? For the purpose of this research, the literature review was focused on the perceptions of healthcare clinicians in the primary care setting, as literature was found to be strongest in this area. This study focused on following healthcare clinicians: pediatrician, OBGYN, PCP, advanced practice clinicians. Using the term "PCP", referencing the literature, was applied for the analysis of all focused healthcare clinicians for the purpose of this study.

\section{Literature Review}

\section{Healthcare Clinicians and Well-baby Visits}

Primary care is defined as, "integrated, accessible, and collaborative health care delivered by providers who are responsible for the majority of a patient's health care needs" []]. A PCP's 
role in healthcare is broad, as they treat patients throughout the lifespan. Specifically, pediatricians are PCPs who specialize in treating patients from gestation through young adulthood. OBGYNs typically treat patients from adolescence through adulthood. However, OBGYNs are inadvertently responsible for gestational and baby welfare as they focus on maternal health from pregnancy, which is inclusive of the 22 week perinatal phase [1]. Advanced practice clinicians encompass both physician assistants and nurse practitioners. For these reasons, pediatricians, OBGYNs, and other advanced practice clinicians are all considered perinatal specialists and are responsible for well-baby visits during the perinatal and infancy periods.

A well-baby visit typically includes comprehensive physical exams, immunizations, and developmental screenings. During well-baby visits, parents and caregivers are counseled by a PCP on issues relevant to maternal and infant health $[\underline{10}]$. PCPs are currently experiencing workforce shortages, limited availability, and a lack of resources, which could be mitigated by introducing interprofessional care [7]. Current research conducted in the form of focus groups indicated that some parents are already reliant on the healthcare clinician to initiate conversations about development, rather than bringing up topics themselves [] $]$. For example, Krantz, Gievers, and Khaki [11] conducted a retrospective study in which the medical records of 300 newborns were analyzed in an effort to identify the frequency of varying concerns among parents during their well-baby visits. The study concluded that the most common themes of concern were in terms of feeding, jaundice, and sleep. However, there was no way to account for questions or concerns that the parents felt uncomfortable asking during these visits [11].

\section{Prevention}


As stated previously, parents and caregivers have reported a lack of understanding of developmental milestones, limited time with their clinician, and a lack of resources provided during their periodical well-baby visits $[\underline{2}, \underline{6}, \underline{11}]$ This lack of understanding is further supported in the literature with a study by Zachary and Kitzmann [12] on parents and caregivers' awareness of the importance of "tummy time," or prone play. The researchers examined where the parents and caregivers received information (e.g. hospital staff, pediatrician, printed materials, friends or family, media, nurse, or other). Their results found that one-fourth of parents and caregivers were not aware of prone play recommendations and of those parents and caregivers, 25 percent did not know the potential complications of development that could occur without prone play [12]. These complications could include plagiocephaly, delay of developmental motor milestones, and decreased head and neck control. This indicates that there are still many parents and caregivers that remain unaware of practices that promote infant development, despite current parental guidance efforts [] $]$. Other services, such as OT, may be warranted to provide a reliable and direct source of information to address concerns such as plagiocephaly and motor development.

A common theme found in the literature was that parents and caregivers are receptive to learning about child development [13-16]. Researchers Parush and Hahn-Markowitz [15] conducted a study of an early prevention program where mothers help their infants' development in the first couple months after birth. Their study described becoming a parent or caregiver as instinctual, but stated that there is still the need for education and knowledge to aid in their child's development. This study concluded that all of the subjects' developmental knowledge increased, as well as their ability to influence their child and apply the knowledge gained from the early prevention program into practice; in comparison to those who were not involved in the intervention of the program. 
A wealth of research reports that parents and caregivers prefer a hands-on approach during scheduled visits $[\underline{17}, \underline{18}]$. Parents and caregivers believe that face-to-face interactions allow for more effective communication and understanding of preventative techniques. Potential miscommunication between PCPs and parents and caregivers can result in negative effects, such as overfeeding or decreased compliance with safe sleeping position guidelines [18]. Based on this evidence, it can be concluded that learning is multifaceted and information should therefore be presented to accommodate various learning styles [17]. Development and feeding are both areas of expertise for OTPs []ㅡ. This expertise further supports the need for OTPs involvement within the primary care setting in order to address various parental learning styles.

\section{Occupational Therapy}

As a meritable solution to address the lack of parental education, decreased time availability for PCPs, and the minimal ability to delegate this education to other healthcare providers, this research question proposes the involvement of OTPs during well-baby visits $[\underline{2-6}, \underline{8}, \underline{15}, \underline{18}]$. Cognitive development, mental health, motor development, self-help, social participation, and rest and sleep are included within the pediatric OT scope of practice [19]. Services include identifying occupational needs, family education, environmental adaptations, and intervening within natural context and routine. Current OT pediatric practice areas include inpatient hospital settings, outpatient hospital settings, community settings, and home based settings such as EI [19]. OTPs offer a holistic perspective within these practice areas by using models from health promotion theories for pediatric patients. Reitz and Scaffa [20] defined health promotion as "improved health and well-being, quality of life, and participation for individuals, families, and populations" $[\underline{20}$ p. 2]. The focus of the health promotion model includes prevention in the incidence of illness and secondary conditions or disease, promotion of 
positive mental health and healthy living practices, and risk reduction strategies throughout the lifespan. The health promotion approach relates to OT and primary care as a preventative measure for developmental delay.

\section{Occupational Therapy within Primary Care}

Evidence supports that primary care is an excellent emerging practice area for OTPs to implement parent education and assess developmental milestones. Roberts, Farmer, Lab, Muir, and Siebert [21] described the correlations between primary care and OT by explaining how OTPs have an "educational background in the liberal arts as well as biological, physical, social, and behavioral sciences," [21 p. S26], much like PCPs. This educational background of OTPs supports client-centered care and occupational engagement. OTPs contribute to primary care practice with specific aims that include prevention of secondary complications, health promotion, family and caregiver assistance and support, and self-management. Researchers, Winship, Ivey, and Etz [22] , identified the needs and roles of OTPs in a primary care setting by conducting clinician and patient interviews for two months in which patients reported their experiences, perceived health changes, and challenges to occupational performance. Results showed that all participants indicated deficits with patient resources, medical management, mental health issues, and occupational challenges. The authors concluded that primary care patients have occupational needs that are not always apparent to clinicians.

Interprofessional collaboration and communication is the key to bridging the gaps that exist with OTPs, PCPs, and parents. Interprofessional involvement has the potential to extend face-to-face interaction between parents and practitioners. A lack of understanding of OT as a profession was identified as a barrier by PCPs. Another barrier was that other team members did not accept OTPs into PCP offices. To address these barriers, OTPs can collaborate and educate 
the staff through staff meetings, hallway consults, or informal presentations in order to share their expertise and express involvement in each patient's care [233, 24]. PCPs' unfamiliarity with OT practice and how it can benefit their patients leads to underutilization of OT services [24]. Interprofessional collaboration has occurred with PCPs and OTPs conducting assessments and interventions together with the patient and by delegating non-physician tasks to the OTP, such as documentation, education or counselling, and developmental screenings prior to physical exams $[\underline{23}, \underline{24}]$.

The evidence undeniably shows the need and efficacy of OT services as a part of the interprofessional team in the primary care setting. Coordinating care as a team will optimize infant development and prevent secondary complications. OT services within the primary care setting will also improve access to therapy services for patients who would otherwise not have access to the desired level of care [25]. It would be the OTPs' responsibility to ensure that other interprofessional team members understand their scope of practice by providing education during daily interactions in addition to providing education to patients during the well-baby visit [르].

\section{Early Intervention Services}

EI services primarily provide therapeutic interventions to patients from birth to age three, which is inclusive of the perinatal and infancy periods. The plasticity of the brain is greatest during the perinatal period. Therefore, it is essential for parents to seek care from developmental specialists as soon as possible [27]. Neuroplasticity is the brain's ability to redirect neural pathways in response to new experiences. Neuroplastic properties in the brain have been found to decrease with age, which highlights the importance of early recognition and referral to EI services during a child's first well-baby visit [료]. 
In order to enhance the parent-child relationship, some EI therapists have identified a need to focus on interventions that are participation-based; meaning that the family or caregivers play an active role during therapy [29]. It is argued that the active, hands-on approach allows for improved learning based on opportunity for practice and feedback for techniques to carry over when the therapist is not present [29]. A study compared the perceptions of 55 mothers of infants receiving EI to a control group of 54 mothers whose infants were not receiving EI over an eight week period [15]. The study found that the intervention group demonstrated increased knowledge and understanding about their child's development without regard to their current formal education level. This study can be used to further support the argument that primary care offices would benefit from the inclusion of OTPs during patient education about development during well-baby visits [토].

It can be inferred from previous literature that there is a need for more effective methods of education within primary care that relates to the perinatal and infancy periods; particularly related to the health promotion model and preventive developmental guidance. OTPs can supplement the resources currently provided by PCPs to achieve better parental understanding of infant development. The abundance of parental resources and face-to-face interactions with health care practitioners can enhance this effort by supporting interprofessional communication within an infant's health care team. This research foresees a future in integration of OT within the primary care setting for more reliable and effective care for infants and their families throughout critical developmental stages by gathering the current perceptions of PCPs and studying their potential impact on OTP involvement.

The purpose of this study was to determine the perceptions of healthcare clinicians on having OTPs provide preventative education and developmental guidance to parents or 
caregivers during their scheduled well-baby visits. The objective of this research was to gather data on the perceptions of healthcare clinicians' understanding of the OT scope of practice, specifically, how OT can be integrated into the primary care setting. It was hypothesized that healthcare clinicians would be receptive to having OTPs help assess developmental milestones and provide preventative and developmental guidance to parents and caregivers during well-baby visits.

\section{Methods}

This study collected data through the use of a survey to analyze the perceptions of healthcare clinicians on the integration of OTPs during a well-baby visit for the purpose of assessing developmental milestones and providing parental guidance. The survey, Perceptions of Healthcare Clinicians on Occupational Therapists Providing Services During Well-Baby Visits, was distributed to an accessible population of licensed practitioners.

\section{Subjects}

The target population for this research included all current and retired clinicians. This population was limited by a convenience sample based on those who self-selected to participate in the survey within Facebook groups. This research was inclusive of all genders, races, ethnicities, and years of professional experience. For the purpose of this study, healthcare clinicians were inclusive of PCPs, pediatricians, OBGYNs, and advanced practice clinicians. Additional inclusion criteria encompassed those who have access to the technology needed to complete the survey. Not all of the questions on the survey were accessible to screen-reading programs, leading to a possible exclusion of individuals who require assistance.

This study aimed for a sample size between 400-500 participants. The sample size was chosen based on significance found in previous literature using similar measurement tools 
$[\underline{2}, \underline{8}, \underline{10}]$. This research aimed to have a return rate of 200 completed surveys through voluntary participation.

\section{Materials}

Data was collected using a survey from Qualtrics XM platform through Moravian College. Demographic questions of gender, race, ethnicity, and years of professional experience were asked using multiple choice selection (see Appendix) in order to ascertain if the returned sample is representative of the targeted population of healthcare clinicians. Likert scale questions were utilized to collect information regarding the understanding of the occupational therapy scope of practice and the perceptions of parental education. Participants were asked to report their perceptions on the Likert scale with five selections ranging from disagree (1) to agree (5) (see Appendix). Before distributing the Qualtrics survey to the sample population, face and content validity of the survey was assessed by receiving feedback from two pediatricians, two OBGYNs, and two advanced practice clinicians.

\section{Procedures}

The vetted survey was distributed by the research team to clinicians using a convenience sample through Facebook groups, where subjects were self-selected through non-probability sampling (see Appendix). Subject self-selection was chosen to reduce probability of invalid data, as internet surveys have been found to increase participant comfortability compared to face-to-face methods [일 . If the intended sample size was not met, a snowball recruitment approach will be utilized to increase response rates.

Before beginning the survey, informed consent was obtained within the Qualtrics XM platform. Through the informed consent, clinicians were notified of potential risks involved by participating in the survey. Sharing information via the internet poses a risk for data hacking, 
however, this risk was reduced by collecting the data through the encrypted Qualtrics XM platform for security purposes. No information related to the clinicians' names or contact information was linked to the research data. Survey respondents were asked to select their professional title to ensure all clinicians met the inclusion criteria. If inclusion criteria was not met, participants were not able to answer the proceeding questions. The subjects' selections remained protected through Qualtrics' privacy settings to ensure the results remain anonymous.

\section{Data Analysis}

The perceptions of healthcare clinicians were analyzed by researchers as quantitative data from the Likert scale ratings. The Likert scale ratings were coded as follows: Disagree $(D)=1$, Somewhat disagree $(S D)=2$, Neutral $(N)=3$, Somewhat agree $(S A)=4$, Agree $(A)=5[\underline{31}]$. The quantitative data was entered into the Statistical Package for the Social Sciences (SPSS) analytic software. The SPSS software was used to run a non-parametric Spearman correlation. In addition to the analysis of the data, researchers anticipated performing intergroup analyses to explore relationships between perceptions and varying demographic data. All surveys and data were removed from the Qualtrics XM platform once data has been analyzed.

\section{Results}

\section{Participants}

A total of 95 participants recruited through Facebook groups and snowball sampling answered the elective Qualtrics survey. Demographics of the clinicians are displayed in Table 1.1 and Figure 1.1. It is notable that the majority of clinicians identified themselves as white (85) and non-hispanic (87). Advanced practice clinicians were the majority of the participants, followed by pediatricians, OBGYNs, and PCPs (see Figure 1.1). 
The non-parametric Spearman correlation was the primary analysis chosen to analyze the relationship between gender, professional title, and years of experience with the perceptions of OT inclusion in a well-baby visit. The Shapiro-Wilks Test of Normality resulted in a value of $\mathrm{p}<0.05$, indicating that the data set was not normally distributed and met all of the other assumptions to run the Spearman correlation [31].

The results aimed to answer the following research question: What are the perceptions of healthcare clinicians (PCPs, pediatricians, OBGYNs, and other advanced practice clinicians) regarding OTPs providing preventative education and developmental guidance to parents and caregivers during their scheduled well-baby visits? The hypothesis assumed that healthcare clinicians would be receptive to having OTPs included in a well-baby visit. Table 1.2 shows the results of the Spearman correlation analysis which indicated that gender, professional title, and years of experience had no correlation with the perceptions of clinicians on the inclusion of OTPs in the primary care setting $(\mathrm{p}>0.05)$.The Likert scale question, "I believe that having occupational therapists provide preventative education and developmental guidance to parents and caregivers during perinatal visits would be beneficial in preventing developmental delay," was analyzed as the primary analysis because it most directly targets the research question and seeks to prove the hypothesis.

There was not a significant correlation between the variables analyzed, therefore the null hypothesis is accepted. However, based on this study's response rate, there is evidence that 75 healthcare clinicians would be receptive to having OTPs help assess developmental milestones and provide preventative and developmental guidance to parents and caregivers during well-baby visits (see Table 1.3). 
Analysis on the seven remaining Likert scale questions were compared to help determine the perceptions of clinicians on various topics (i.e. parent education, time, knowledge of OT). Similar questions were grouped to visually compare the majority of responses that were selected based on the Likert scale (Figures 1.2, 1.3, 1.4). Figure 1.2 refers to the survey prompt, "There is sufficient time allotted for providing parental education," where a majority of clinicians disagreed or somewhat disagreed with the statement (56;58.9\%) (see Figure 1.2). Figure 1.3 shows a comparison between four survey prompts where clinicians somewhat agreed or agreed with the following: “An adequate amount of parent education is provided to parents during well-baby visits" (64; 67.3\%); "Providing parental education during well-baby visits is a preventative approach to developmental delay" (90; 94.7\%); "Parental figures are seeking more guidance regarding their child's developmental milestones" (68; 71.6\%); "Adequate resources are provided to patients and their families in order to address developmental and parental concerns" (52; 54.7\%). Figure 1.4 shows that the majority of clinicians somewhat agreed or agreed with the survey prompt, "I am familiar with occupational therapy and understand their scope of practice" (78;82.1\%) and "I am aware that occupational therapists are trained as developmental specialists" (72; 75.8\%).

\section{Discussion}

When considering the clinical implications of the results, the research indicated that the majority of healthcare clinicians would be receptive to having OTPs involved as developmental specialists during well-baby visits. This result is impactful on OT practice and can be used as introductory evidence to support the idea that primary care is an emerging practice area for OTPs working alongside physicians and advanced practice clinicians in the realm of primary care pediatrics and OBGYNs' perinatal realm. Three themes were identified from the remaining 
Likert scale questions: there is insufficient time during well-baby visits, there is not enough resources or education provided to parents during well-baby visits, and OT services are understood by healthcare clinicians.

These themes are congruent to the themes found within the previous literature. Time constraints related to physician productivity and face-to-face patient interaction has been identified as a key limitation in prevention of developmental delays throughout the literature [ $\underline{2}$, $\underline{6}, 11]$. Results of this study are similar to literature findings, as the majority of respondents stated that limited time has been apparent in well-baby visits. This supports Lipkin et al.'s [2] research that lack of time during well-baby visits is a significant factor in prevention of development delay.

Despite the findings found in the literature that parents do not feel they are receiving an adequate amount of resources and education during well-baby visits, this research found that healthcare clinicians perceive that they are providing enough education and resources to parents and caregivers [15]. OTPs in this emerging practice area will be challenged with bridging the gap between the perceptions of healthcare clinicians and the perceptions of parents as part of their role in primary care.

This research found that the majority of healthcare clinicians agree that parents are seeking more guidance in regard to developmental milestones. This finding is supported by previous literature by Zachary and Kitzmann [12], where it was found that $25 \%$ of parents were unaware of developmental complications such as plagiocephaly, delay of developmental motor milestones, and decreased head and neck control that can result from not understanding or adhering to prone-play recommendations. 
The findings by Winship, Ivey, and Etz [ㄹ2] recognized that primary care patients have occupational needs that are not always apparent to clinicians. However, clinicians also understood the OT profession and OTPs' role as developmental specialists [22]. Based on these conflicting findings, it can be challenging for OTPs to identify the need for peer education on the OT scope of practice and avoid the underutilization of OT services [24].

\section{Limitations}

Although 95 clinicians were able to complete the survey, it did not meet the goal to receive 200 completed survey responses. In addition, the survey samples were not evenly distributed among professions and genders. This uneven distribution may have provided biased responses. For example, the majority of survey respondents were advanced practice clinicians, resulting in their perceptions having a greater influence on the outcomes of the study. Additionally, the methods of convenience sampling and snowball recruitment may have also restricted the sample demographics, as demonstrated by more female respondents than males. However, it is notable that the workforce distribution is mainly female and advanced practice clinicians encompasses multiple areas of practice. Another limitation was the inability to measure the integrity of the clinicians' responses. The survey did not gather qualitative evidence that would confirm accurate understanding of the topic being measured. For example, there is no way to justify that the clinicians who participated in the study have an accurate understanding of the scope of OT.

\section{Future Direction}

There is limited information available that links benefits of including OTP in perinatal services and pediatrics within the area of well-baby visits. The literature found on the inclusion of OTPs in the perinatal realm and primary care indicates a need for advocacy efforts to include 
OTPs within primary care by focusing on the benefits and special skills that OTPs can provide, as well as advocacy efforts for clinicians to include other specialists to address special needs of infants and young children.

Additionally, the theme of insufficient time requires further research to explore the relationship between duration of the well-baby visits and education provided. This theme can focus on identifying which discipline experiences shorter visits, how this impacts the health of children and parents' understanding of their child's health and development, and developing a solution to address various learning styles of parents. Does this current practice of short visits show negative consequences in the overall health and well-being of infants and children? How can OTPs relieve this strain that exists in primary care and perinatal visits?

Since the results did not yield any statistically significant correlations between the variables (gender, professional title, years of experience) with perceptions, further research may be appropriate to explore what impacts clinicians' perceptions. Is it knowledge disparities or lack of prior experience with occupational therapy? There are also opportunities to focus future research on a singular demographic variable (i.e. race, ethnicity, education) and its impact on the perceptions of clinicians. To address the limitations of this research, interviews or focus groups could be conducted with healthcare clinicians to adequately measure the understanding or perceptions of topics being measured.

\section{Conclusion}

Majority of clinicians believed the inclusion of OT during a well-baby visit would be beneficial to provide preventative education and developmental guidance to prevent developmental delay. This research was successful in determining that gender, age, and years of experience did not influence the perceptions of healthcare clinicians in regard to the inclusion of 
OTPs in primary care. Additional findings indicated that there is a discrepancy between the needs of parents and caregivers and the perceptions of healthcare clinicians regarding the sufficiency of education and resources provided during well-baby visits. These findings are in agreement with previous studies that parents are seeking more resources regarding development and that time is a key influence in determining the amount of education provided during well-baby visits. 


\section{References}

1. World Health Organization. International classification of functioning, disability and health. 2001. https://www.who.int/classifications/icf/icfbeginnersguide.pdf. Accessed 28 May 2020.

2. Lipkin P, Macias M, Chen B, Coury E, Gottschlich E, Hyman S, Sisk B, Wolfe A, Levy S. Trends in pediatric development screening: 2002-2016. Office Journal of the American Academy of Pediatrics, 145(4). 2020; https://doi.org/10.1542/peds.2019-0851

3. Case-Smith J, Frolek Clark GJ, Schlabach TL. Systematic review of interventions used in occupational therapy to promote motor performance for children ages birth-5 years. American Journal of Occupational Therapy. 67(4):413-424. 2013; https://doi.org/10.5014/ajot.2013.005959

4. Halfon N, Stevens GD, Larson K, Olson LM. Duration of a well-child visit: Association with content, family-centeredness, and satisfaction. Pediatrics, 128(4), 657-664. 2011; https://doi.org/10.1542/peds.2011-0586

5. Shannon P. Barriers to family-centered services for infants and toddlers with developmental delays. Social Work, 49(2), 301-308. 2004; https://doi.org/10.1093/sw/49.2.301

6. Radecki L, Olson LM., Frintner, MP, Tanner JL, Stein MT. What do families want from well-child care? Including parents in the rethinking discussion. Pediatrics, 124(3), 858-865. 2009; https://doi.org/10.1542/peds.2008-2352

7. Jordan K. Occupational therapy in primary care: Positioned and prepared to be a vital part of the team. American Journal of Occupational Therapy, 73(5). 2019; https://doi.org/10.1542/peds.2008-2352 
8. American Occupational Therapy Association. Occupational therapy practice framework: Domain and process. American Journal of Occupational Therapy, 68(Suppl. 1), S1-S48. 2014; https://doi.org/10.5014/ajot.2014.682006

9. Unanue R, Westcott S. Neonatal asphyxia. Infants \& Young Children: An Interdisciplinary Journal of Early Childhood Intervention, 13(3), 13-24. 2001. https://doi-org.moravian.idm.oclc.org/10.1097/00001163-200113030-00006

10. Hemmelgarn BR, Edouard L, Habbick BF, Feather J. Duplication of well-baby services. Canadian Journal of Public Health. 1992. 83(3): 217-220.

11. Krantz J, Gievers L, Khaki S. Investigating parental concerns at the first well-child visit. Clinical Pediatrics, 59(1), 83-86. 2020; https://doi.org/10.1177/0009922819884571

12. Zachary AH, \& Kitzmann KM. Caregiver awareness of prone play recommendations. American Journal of Occupational Therapy, 65(1),101-105. 2011; https://doi.org/10.5014/ajot.2011.09100

13. Goldstein LA. Family support and education. Physical \& Occupational Therapy in Pediatrics, 33(1), 139-161. 2013; https://doi.org/10.3109/01942638.2012.754393

14. Hickey, G., McGilloway, S., Leckey, Y., \& Stokes, A. (2018). A universal early parenting education intervention in community-based primary care settings: development and installation challenges. Education Sciences, 8(4), 178-178. https://doi.org/10.3390/educsci8040178

15. Parush S, Hahn-Markowitz J. The efficacy of an early prevention program facilitated by occupational therapists: a follow-up study. The American Journal of Occupational Therapy : Official Publication of the American Occupational Therapy Association. 1997. 51(4): 247-251. 
16. Shah R, DeFrino D, Kim Y, Atkins M. Sit down and play: A preventive primary are-based program to enhance parenting practices. Journal of Child \& Family Studies, 26(2), 540-547. 2017; https://doi.org/10.1007/s10826-016-0583-6

17. McMahon SE. Enhancing motor development in infants and toddlers: A Multidisciplinary process for creating parent education materials. Newborn \& Infant Nursing Reviews, 13(1), 35-41 2013; https://doi.org/10.1053/j.nainr.2012.12.001

18. Gazmararian J, Dalmida S, Merino Y, Blake S, Thompson W, Gaydos L. What new mothers need to know: Perspectives from women and providers in Georgia. Maternal \& Child Health Journal, 18(4), 839-851. 2014; https://doi.org/10.1007/s10995-013-1308-8

19. Clark G, Kingsley K. Occupational therapy practice guidelines for early childhood: Birth-5 years. American Journal of Occupational Therapy, 74(3). 2002; https://doi.org/10.5014/ajot.2020.743001

20. Reitz M, Scaffa M. Occupational Therapy in the Promotion of Health and Well-Being. American Journal of Occupational Therapy, 74(3). 2020; https://doi.org/10.5014/ajot.2020.743003

21. Roberts R, Farmer M, Lamb A, Muir S, Siebert C. The role of occupational therapy in primary care. American Journal of Occupational Therapy, 68, S25-S33. 2014; https://doi.org/10.5014/ajot.2014.686S06

22. Winship J, Ivey C, Etz R. Opportunities for occupational therapy on a primary care team. The American Journal of Occupational Therapy, 73(5). 2019; https://doi.org/10.5014/ajot.2019.030841

23. Dahl-Popolizio S, Muir SL, Davis K, Wade S, \& Voysey R. Occupational therapy in primary care: Determining receptiveness of occupational therapists and primary care 
providers. Open Journal of Occupational Therapy (OJOT), 5(3), 1-12. 2017; https://doi.org/10.15453/2168-6408.1372

24. Donnelly C, Brenchley C, Crawford C, Letts L. The integration of occupational therapy into primary care: A multiple case study design. BMC Family Practice, 14(1), 60-71. 2013; https://doi.org/10.1186/1471-2296-14-60

25. Donnelly, C. A., Brenchley, C. L., Crawford, C. N., \& Letts, L. J. The emerging role of occupational therapy in primary care. Canadian Journal of Occupational Therapy. 81(1), 51-61. 2014. https://doi-org.moravian.idm.oclc.org/10.1177/0008417414520683

26. Halle AD, Mroz TM, Fogelberg DJ, Leland NE. Occupational therapy and primary care: Updates and trends. American Journal of Occupational Therapy, 72(3): 7203090010. 2018; https://doi.org/10.5014/ajot.2014.686S06

27. Mundkur N. Neuroplasticity in children. The Indian Journal of Pediatrics, 72(10), 855-857. 2005; https://doi.org/10.1007/BF02731115

28. Guyer AE, Pérez EK, Crone EA. Opportunities for neurodevelopmental plasticity from infancy through early adulthood. Child Development, 89(3), 687-697. 2018; https://doi.org/10.1111/cdev.13073

29. Colyvas JL, Sawyer LB, Campbell PH. Identifying strategies early intervention occupational therapists use to teach caregivers. American Journal of Occupational Therapy, 64, 776-785. 2010; https://doi.org/10.5014/ajot.2010.09044

30. De Leeuw E. Self-administered questionnaires and standardized interviews. In Alasuutari P, Bickman L, Brannen J. The SAGE handbook of social research methods (pp. 313-327). London: SAGE Publications Ltd. 2008; http://dx.doi.org/10.4135/9781446212165.n18 
31. Portney LG, Watkins PM. Foundations of Clinical Research: Applications to Practice.

3rd ed. Philadelphia, PA: FA Davis; 2015. 


\section{Figures}
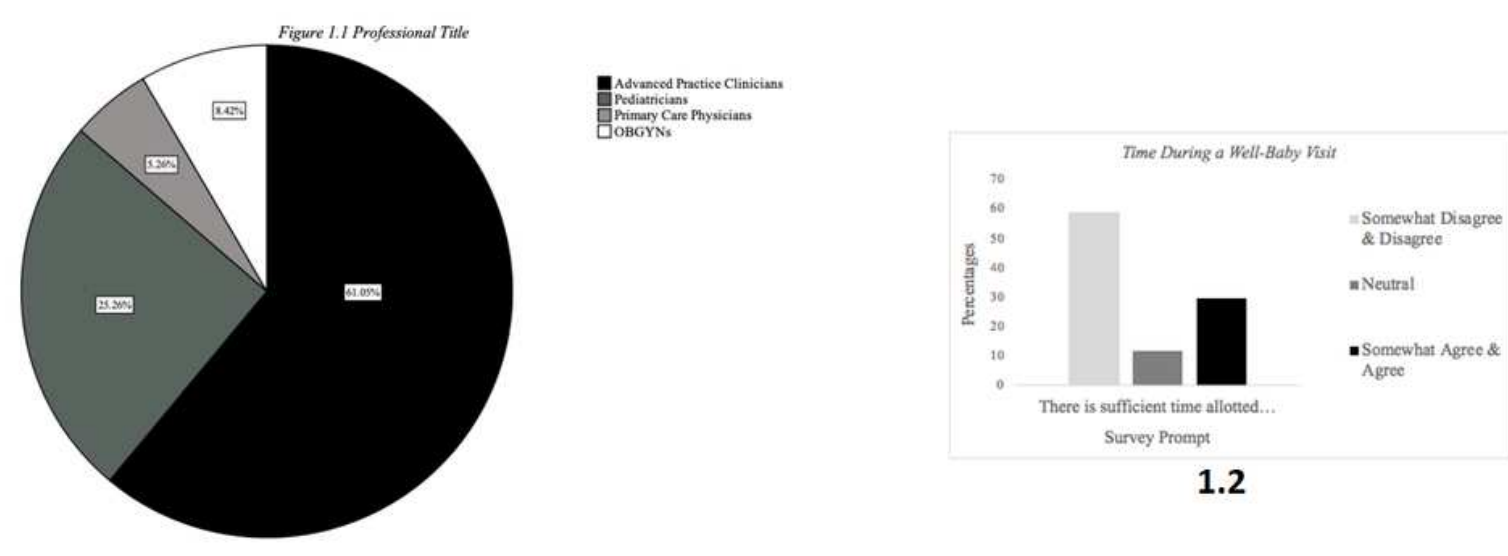

1.2

1.1
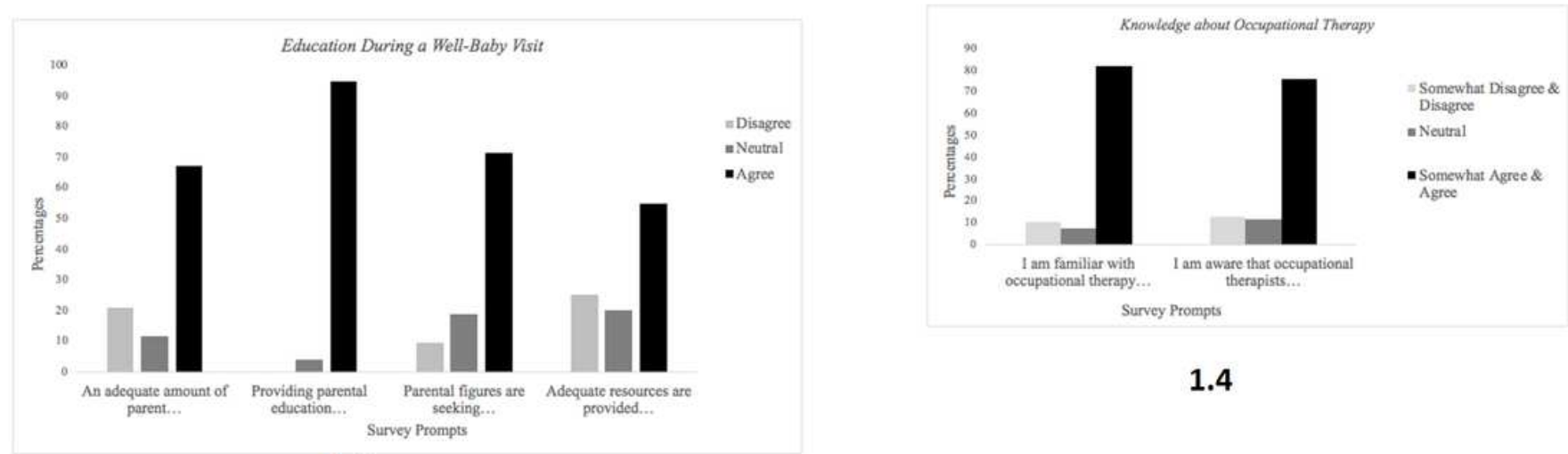

1.4

1.3

\section{Figure 1}

1.1. Compares the professional titles of the surveyed clinicians. 1.2. Majority of clinicians either disagree or somewhat disagree with the survey prompt, "There is sufficient time allotted to providing parental education" (58.9\%). 1.3. Majority of clinicians somewhat agreed or agreed with the following survey prompts: "An adequate amount of parent education is provided to parents during well-baby visits" (67.3\%); "Providing parental education during well-baby visits is a preventative approach to developmental delay" (94.7\%); "Parental figures are seeking more guidance regarding their child's developmental milestones" (71.6\%); "Adequate resources are provided to patients and their families in order to address developmental and parental concerns" (54.7\%). 1.4. Majority of clinicians somewhat agreed or agreed with the survey prompt, "I am familiar with occupational therapy and understand their scope of practice" (82.1\%) and "I am aware that occupational therapists are trained as developmental specialists" (75.8\%). 


\section{Supplementary Files}

This is a list of supplementary files associated with this preprint. Click to download.

- QualtricsSurvey2.pdf

- Table1.11.21.3.docx 